\title{
First Neutrino Oscillation Results from the NOvA Experiment
}

\author{
Kanika Sachdev* \\ Fermilab \\ E-mail: ksachdevefnal.gov
}

NOvA is a long-baseline neutrino oscillation experiment on the NuMI muon neutrino beam at Fermilab. It consists of two functionally identical, nearly fully-active liquid-scintillator tracking calorimeters. The Near Detector (ND) at Fermilab is used to study the neutrino beam spectrum and composition before oscillations occur. The Far Detector in northern Minnesota, $810 \mathrm{~km}$ away, observes the oscillated beam and is used to extract the oscillation parameters. NOvA is designed to observe oscillations in two channels: disappearance channel $\left(v_{\mu} \rightarrow v_{\mu}\right)$ and $v_{e}$ appearance channel $\left(v_{\mu} \rightarrow v_{e}\right)$. This paper reports the measurements of both these channels based on the first NOvA data taken from February 16, 2014 till May 15, 2015.

XIII International Conference on Heavy Quarks and Leptons

22-27 May, 2016

Blacksburg, Virginia, USA

${ }^{*}$ Speaker. 


\section{Introduction}

Flavor oscillations of neutrinos have been observed in neutrinos from solar [1], atmospheric [2], accelerator [3] and reactor [4, 5, 6] sources, and the non-zero mass of neutrinos firmly established. The dynamic phase in the oscillation probability of neutrinos depends on the $\mathrm{L} / \mathrm{E}$ ratio for an experiment, where $\mathrm{L}$ is the propagation length of neutrinos or the experiment's baseline and $\mathrm{E}$, the energy of neutrinos, and $\Delta m^{2}$, the mass-squared difference between the neutrino mass states. The amplitude of the oscillation depends on the mixing angles $\theta_{13}, \theta_{23}$ and $\theta_{21}$. NOvA is designed to measure the parameters governing the oscillation in the $v_{\mu} \rightarrow v_{\mu}$ disappearance channel and $v_{\mu}$ $\rightarrow v_{e}$ appearance channel.

To first order, the $v_{\mu}$ disappearance probability is $\mathrm{P}\left(v_{\mu} \rightarrow v_{\mu}\right)=1-\sin ^{2} 2 \theta_{23} \sin ^{2}\left(1.27 \Delta m_{32}^{2} \frac{L}{E}\right)$. $\theta_{23}$ is known to be close to $\pi / 4$ and is the least precisely measured mixing angle. It parameterizes the ratio of $v_{\mu}$ and $v_{\tau}$ contributions in the mass state $v_{3}$. The $v_{\mu} \rightarrow v_{e}$ oscillation probability is small because the leading term contains $\sin ^{2} \theta_{23} \sin ^{2} 2 \theta_{13}$ and $\theta_{13}$ is the smallest of the mixing angles at $\sim 8.5^{\circ}$ [7]. The presence of $\sin ^{2} \theta_{23}$ in the leading term makes this channel sensitive to the octant of $\theta_{23}$. The sub-leading terms in this channel depend on CP violating phase $\delta$ and, due to the matter effect, on mass hierarchy, that is whether $v_{3}$ mass state is heavier than $v_{1}$ and $v_{2}$ (normal hierarchy), or the opposite (inverted hierarchy). The $v_{e}$ appearance probability is enhanced in case of normal hierarchy and suppressed in case of inverted hierarchy. Therefore, $v_{\mu} \rightarrow v_{e}$ oscillation is sensitive to three of the least known parameters that govern neutrino oscillations.

The NOvA experiment consists of two functionally identical detectors located $14 \mathrm{mrad}$ offaxis from the NuMI beam. This off-axis placement causes the neutrino energy spectrum to peak at $2 \mathrm{GeV}$, which is well-matched to the first oscillation maximum for the NOvA baseline. The Near Detector (ND) is located $1 \mathrm{~km}$ from the neutrino source and $100 \mathrm{~m}$ underground. The Far Detector (FD) is on earth surface, at $810 \mathrm{~km}$ from the neutrino source in northern Minnesota, and is $14 \mathrm{kT}$ in mass. The detectors consist of extruded PVC cells of cross-section $4 \mathrm{~cm} \times 6 \mathrm{~cm}$. The cells are filled with liquid scintillator and a loop of optical fiber that transports scintillation light to one pixel of a 32-pixel array of Hamamatsu Avalanche Photodiode (APD). The cells are $\sim 4 \mathrm{~m}$ long in the ND and $\sim 15 \mathrm{~m}$ long in the FD. They are arranged in planes in vertical and horizontal orientations, providing top and side view images of particle trajectories in the detector, which enables 3-dimensional reconstruction. More information about the NOvA detector can be found in [8].

The NuMI beam is made by colliding $120 \mathrm{GeV}$ protons on a graphite target, which produces pions and kaons. These decay in a $675 \mathrm{~m}$ long, Helium filled decay pipe, into predominantly $v_{\mu}$ 's $\left(\pi^{+} \rightarrow \mu^{+}+v_{\mu}\right)$. The muons and other hadrons are absorbed in the rock and hadron absorbers, resulting in a $v_{\mu}$ beam. In the range of $1-3 \mathrm{GeV}$ of neutrino energy, the beam contains of $1.8 \% \bar{v}_{\mu}$ and $0.7 \%\left(v_{e}+\bar{v}_{e}\right)$. The results reported here are based on data taken from February 16, 2014 till May 15,2015 , during which time, the FD was exposed to $3.45 \times 10^{20}$ protons on target (POT). The FD was under-construction for some part of this period, and only partially capable of data-taking. Therefore, the full $14 \mathrm{kT}$ equivalent exposure of the FD for this dataset was $2.74 \times 10^{20}$, which is $\sim 7.6 \%$ of the full planned exposure of the NOvA experiment.

The neutrino beam arrives in $10 \mu \mathrm{s}$ long pulses that are $1.33 \mathrm{~s}$ apart. The NOvA timing system 

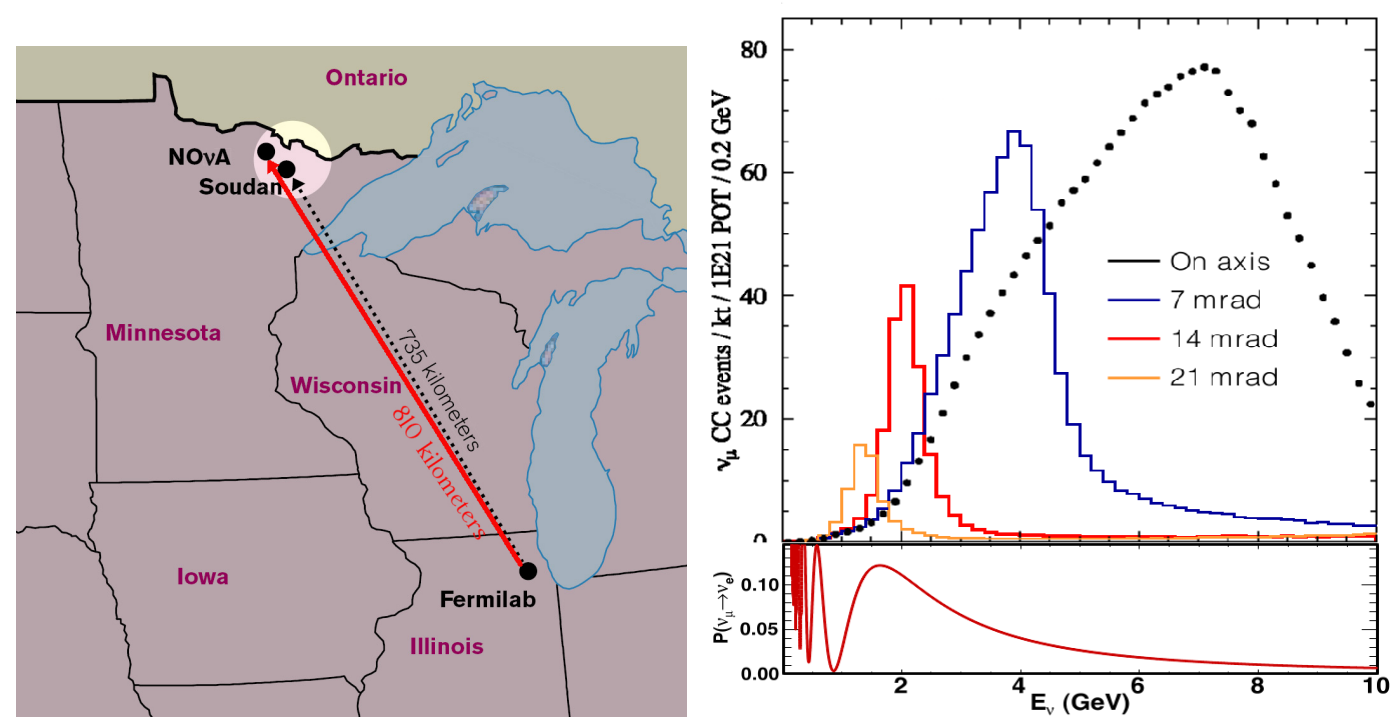

Figure 1: The map shows the location of the NOvA detectors. At the off-axis position of the detectors, the beam energy peaks around $2 \mathrm{GeV}$ which is well-matched to the first oscillation maximum in the $v_{\mu} \rightarrow v_{e}$ transition. Moreover, the beam is much narrower in comparison to the on-axis spectrum. This reduces the neutral current background that feeds down from higher energies.

receives messages broadcast by the Accelerator Division and the data aggregated over a $550 \mu \mathrm{s}$ window, containing the $10 \mu \mathrm{s}$ beam pulse, is written out. The timing side band in these $550 \mu \mathrm{s}$ data windows, outside of the beam pulse, is used to estimate the cosmogenic background to the oscillation analyses.

The oscillation analysis techniques are optimized on a combination of simulation and ND data. The neutrino beam flux is simulated using the FLUGG package, that uses the interaction modeling of FLUKA [9] and the geometry description of GEANT [10]. This package models the interaction of protons with the target and the subsequent decay of the daughter particles in the decay pipe. The NOvA detector geometry is implemented in GDML. The interaction of neutrino with the detector is simulated using GENIE [11] event generator. The energy depositions of the particles produced in neutrino interactions are simulated using GEANT4. Finally, experiment-specific simulation models the scintillation, propagation of photons in the fibers and readout electronics response [12].

The reconstruction and calibration techniques are applied identically to the data and simulation. Hits in the detectors that are spatially and temporally correlated are assumed to arise from the same interaction and are assigned to a reconstructed object called "slice". Further reconstruction is based on hits within a slice and is responsible for reconstructing 3-D tracks or showers corresponding to particle trajectories.

The detectors are calibrated using cosmic ray muons. The raw hits that belong to reconstructed 3-D tracks corresponding to cosmic muons are corrected for light attenuation in the optical fiber and any cell-to-cell variation in performance. The corrected pulses are converted to absolute energy using the minimum-ionization in the last but one meter of the stopping muon tracks as the standard candle. The calibrated energy response is cross-checked using other standard candles such as the $\pi^{0}$ mass peak and the Michel electron energy spectrum. 


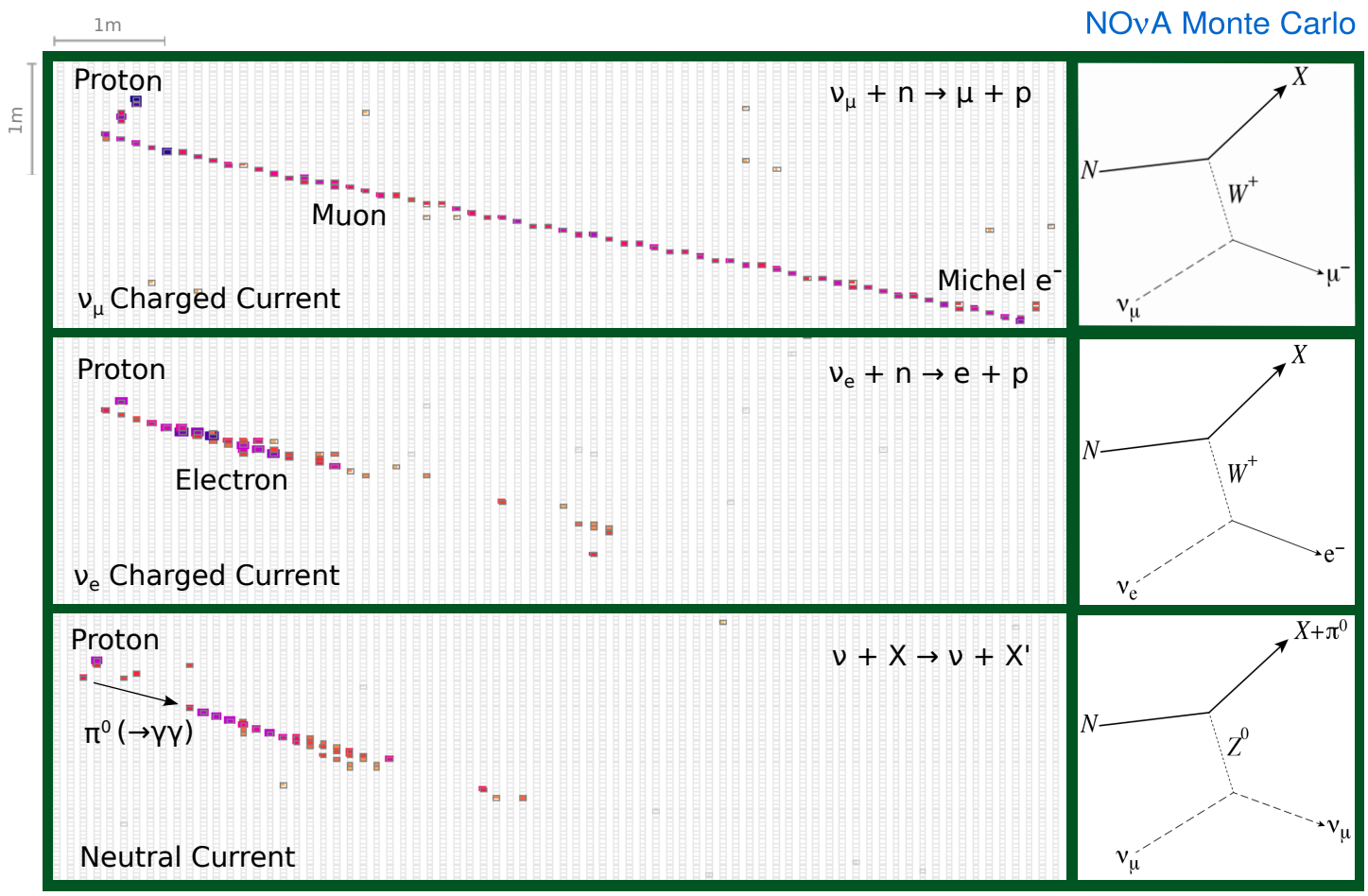

Figure 2: Characteristics of different types of neutrino interactions in the NOvA detectors. $v_{\mu}$ Charged Current (CC) interactions contain a long muon track, along with some hadronic activity. $v_{e} \mathrm{CC}$ interactions have an electron shower instead of a track. Neutral Current (NC) interactions contain no primary charged lepton in the final state.

\section{2. $v_{\mu}$ Disappearance Analysis}

The signal event for this analysis is the $v_{\mu}$ Charged Current (CC) interaction that contains a muon track in the final state, as shown in Figure 2. To identify $v_{\mu}$ CC interactions, a k Nearest Neighbor $(\mathrm{kNN})$ algorithm is employed. The $\mathrm{kNN}$ is trained on variables such as distribution of energy per pathlength along the track $(\mathrm{dE} / \mathrm{dx})$, length of the track, distribution of scattering angles along the track and fraction of planes in the track that contain hadronic energy. The distribution of this particle identifier (PID) on the ND data and MC is shown in Figure 3. It is able to identify $v_{\mu}$ CC interaction with an efficiency of $80 \%$ and a purity of $98 \%$. The rejection of cosmogenic backgrounds in the FD requires additional cuts. A Boosted Decision Tree (BDT) that uses reconstructed quantities such as number of hits, candidate muon track length and direction etc, along with event containment cuts have been designed for this purpose. These cuts, combined, suppress cosmic backgrounds by a factor of $10^{7}$, and the expected rate is estimated at 1.4 events for this analysis.

The energy of the candidate muon is reconstructed from range. The remaining energy in the event is assumed to arise from hadronic recoil and corrected to account for the energy deposited in inactive detector material. The sum of the two is the candidate $v_{\mu}$ energy. When this method is applied in the ND data and MC, the muon energy spectra are found to agree well, however, 

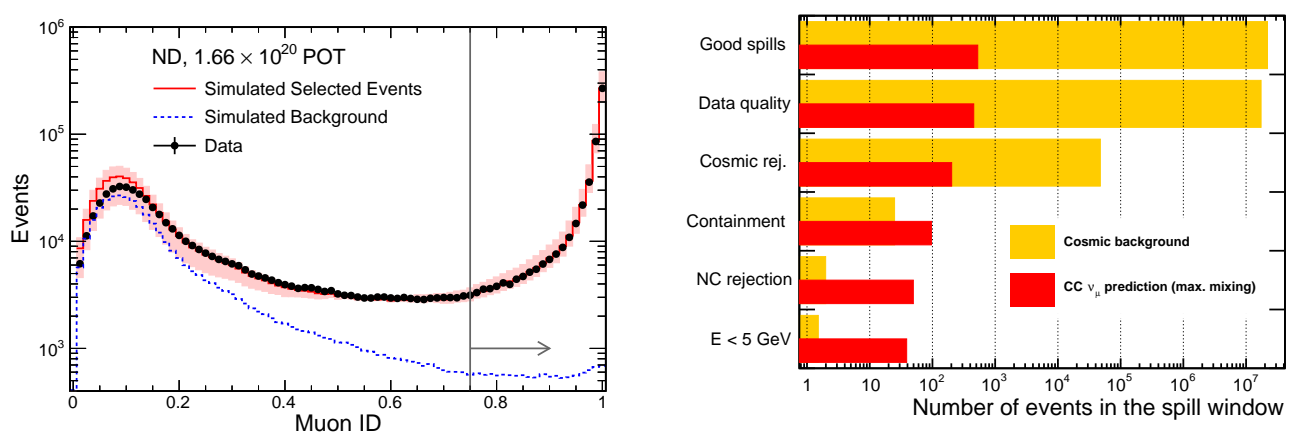

Figure 3: Left: Performance of the $v_{\mu}$ CC PID on the ND data and simulation. Right: The suppression of cosmic background in the FD timing side band data, compared to the $v_{\mu} \mathrm{CC}$ signal.

the hadronic energy spectrum is substantially different between data and MC (see Figure 4). The hadronic response is recalibrated by $14 \%$ to ensure that the reconstructed $v_{\mu} \mathrm{CC}$ energy spectrum matches between data and simulation. The entire size of this recalibration is taken as a systematic on the neutrino energy scale in this analysis.
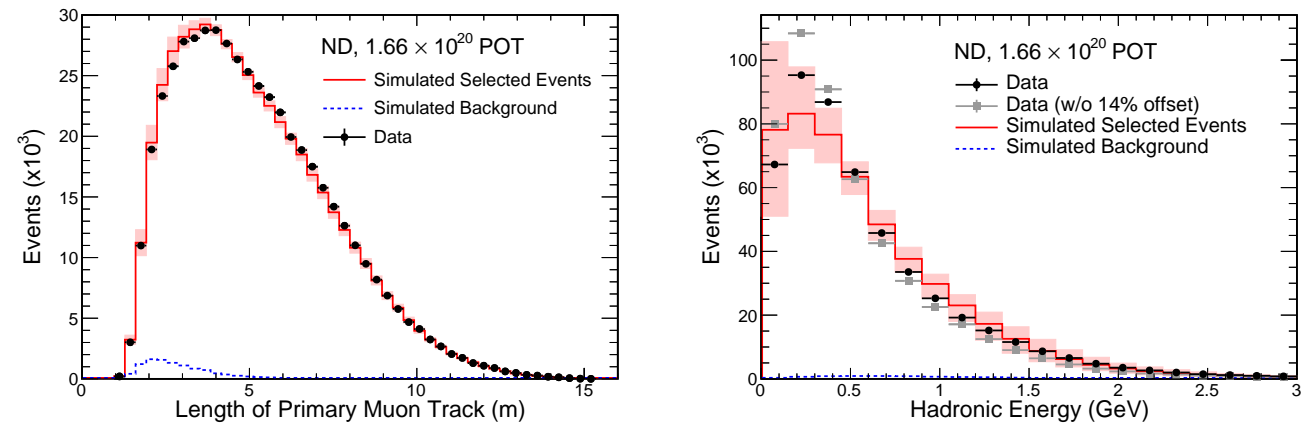

Figure 4: Left: The reconstructed muon energy spectrum agrees well between the ND data and MC. However, the hadronic energy shows a large discrepancy

The ND data reconstructed energy spectrum is converted to a true energy spectrum using a simulation-based migration matrix. The derived true energy spectrum in ND data is corrected by the far to near detector flux ratio from the simulation. This returns a ND data corrected true energy distribution in the FD, which is weighted by the oscillation probability and mapped back to a reconstructed energy spectrum to get the final prediction of $v_{\mu}$ CC disappearance.

Figure 5 shows the expected energy spectrum in the FD if there were no oscillations. The predicted event count in this case is $200.3 \pm 11.9$. However, on applying $v_{\mu} \mathrm{CC}$ selection criteria to the FD data, only $33 v_{\mu}$ CC candidates are selected, which is interpreted as a clear sign of muon neutrino disappearance. The energy spectrum of the data events is described well by the oscillation fit. This result is used to constrain the allowed region in the $\sin ^{2} \theta_{23}$ and $\Delta m_{32}^{2}$ by computing the likelihood difference between the best fit and the oscillated spectra; systematic uncertainties are included as nuisance parameters. Other contours in Figure 5 are results from T2K and MINOS experiments and show that even with this early data, the NOvA measurements are competitive. The best fit for NOvA disappearance measurement described here is: 
normal hierarchy: $\sin ^{2} \theta_{23}$ in the 68\% CL range [0.38, 0.65], $\Delta m_{32}^{2}=+\left(2.52_{-0.18}^{+0.20}\right) \times 10^{-3} \mathrm{eV}^{2}$ inverted hierarchy: $\sin ^{2} \theta_{23}$ in the 68\% $\mathrm{CL}$ range [0.37, 0.64], $\Delta m_{32}^{2}=-(2.56 \pm 0.19) \times 10^{-3} \mathrm{eV}^{2}$ This result has been published in [13].
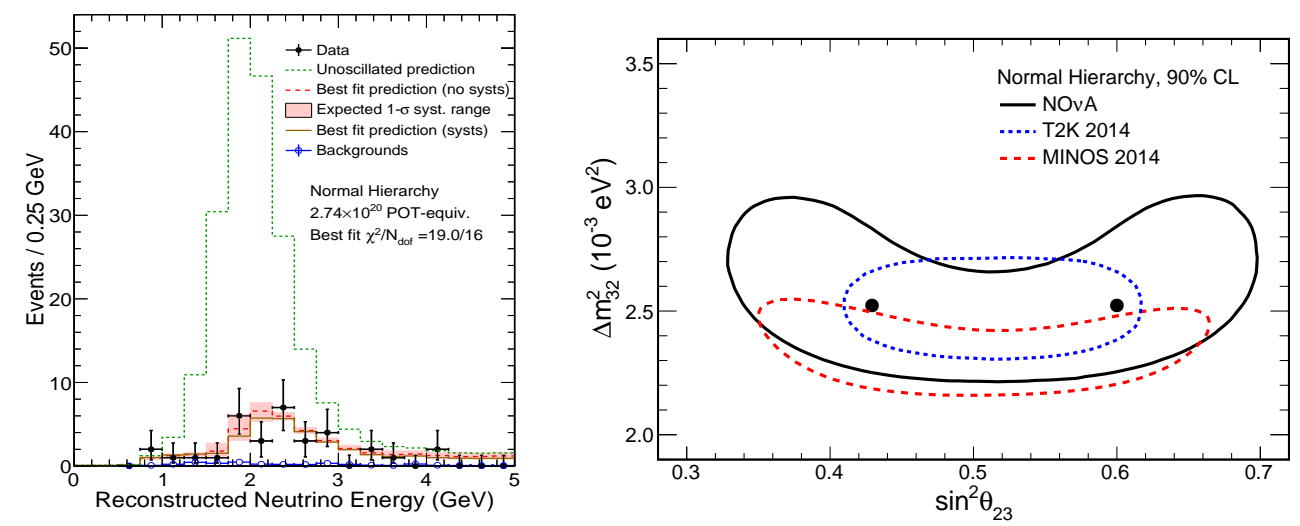

Figure 5: Left: The observed $v_{\mu}$ energy spectrum in the FD data, compared with the no-oscillation prediction. The oscillation fit describes the data very well. Right: The contour shows the region in $\operatorname{the}^{2} \sin _{23}$ and $\Delta m_{32}^{2}$ space allowed at a $90 \%$ confidence level. The NOvA contours are compared with those of T2K and MINOS.

\section{3. $v_{e}$ Appearance Analysis}

The signal for $v_{\mu}$ to $v_{e}$ appearance channel is $v_{e}$ CC interactions in the FD. To select this event type, two PID algorithms have been developed. The primary analysis is done with the Likelihood based PID or LID. In this PID, the longitudinal and transverse $d E / d x$ profiles of the most energetic shower in the event are compared to those of simulated particles and used to construct likelihoods for different particle hypotheses. These, along with other event level information, are used as inputs to an artificial neural network to identify $v_{e} \mathrm{CC}$ events. The secondary analysis is done with Library Event Matching (LEM) PID, where a candidate event is matched against a library of simulated signal and background events. The characteristics of the best matched events are input to a decision tree for $v_{e}$ CC classification. LID is $34 \%$ efficient with respect to contained event sample, while LEM is $35 \%$ efficient. The overlap in the selection by the two PIDs is $62 \%$.

The intrinsic beam $v_{e}$ component of the beam is an irreducible background for $v_{e}$ appearance search. Other backgrounds include neutral current and $v_{\mu}$ CC interactions from the beam and cosmogenic backgrounds. The cosmic and NC backgrounds are dominant at lower event energies while beam $v_{e}$ 's dominate at higher energies. Therefore the energy range of events analyzed for this channel is restricted from 1.5(1.3) to $2.7 \mathrm{GeV}$ for LID (LEM). Further suppression of cosmic background is achieved by imposing strict containment cuts. Cosmic events are vertically oriented, while beam events are close to horizontal in the detector coordinates. Therefore events are also required to be consistent with the beam direction by applying a cut on transverse momentum fraction in the event. The cosmic background is measured in the timing sideband, and is expected to be $\sim 0.06$ event for both, LID and LEM. The rejection of cosmics is shown in Figure 7 . 
Since ND contains no $v_{e}$ appearance signal, all events selected by $v_{e} \mathrm{CC}$ selection in ND are background. The ND simulation is assumed to have the correct proportions of the NC, beam $v_{e}$ $\mathrm{CC}$ and $v_{\mu} \mathrm{CC}$ contributions to the background. The over all normalization is fixed to the ND data. On applying $v_{e} \mathrm{CC}$ selection cuts, $7 \%$ more events are selected in the ND data than simulation, an excess which is assigned equally to the three backgrounds. The $v_{e} \mathrm{CC}$ selected beam background spectra in the FD are corrected by the ratio of the spectra of the backgrounds in the ND data to MC. The signal estimate is constrained by the $v_{\mu}$ CC spectrum in the ND. Selection cuts to select $v_{\mu}$ CC interactions are applied to ND data and simulation. The reconstructed energy spectrum of the the $v_{\mu}$ events in data is mapped to a true spectrum by using a reconstructed to true migration matrix. The true energy spectrum of $v_{e}$ selected events in FD is corrected by the ratio of the true energy spectra of ND data to simulation. The spectra are then multiplied by appropriate oscillation weights to obtain the signal and background estimates. The total number of beam background events expected in FD is 0.94 (1.02) for LID (LEM) and is very loosely dependent on oscillation parameters. In both cases, intrinsic beam $v_{e} \mathrm{CC}$ comprise nearly half the background.
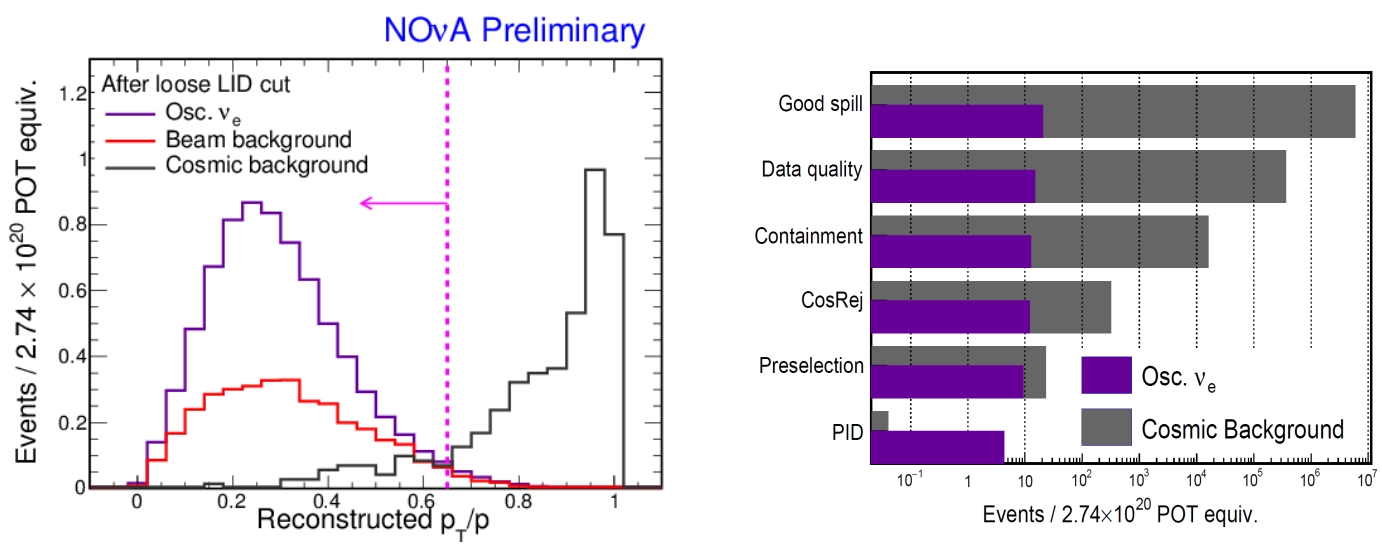

Figure 6: Left: Distribution of LID for $v_{e}$ preselected events in the ND. Right: Reconstructed neutrino energy of LID selected events in ND.

Systematic uncertainties are evaluated by producing systematically shifted samples of simulation in ND and FD, repeating the full analysis with them and computing the change in the expected signal and background counts. Major sources of systematic error in this analysis are neutrino interaction model, energy calibration, scintillator saturation modeling (or Birks' suppression). Because of the two detector configuration of NOvA, flux uncertainties produce a small effect on the oscillation analysis. The total systematic uncertainty on signal for LID (LEM) is 17.6\% (15.0\%) on signal and $10.8 \%(13.4 \%)$ on background.

On applying the $v_{e} \mathrm{CC}$ selection criteria to neutrino beam FD data, we select 6 events with LID. Given the background expectation of 0.99 (beam neutrino + cosmic), this is interpreted as an observation of $v_{\mu} \rightarrow v_{e}$ appearance at $3.3 \sigma$ significance. The LEM PID selects 11 events, which contain the 6 LID-selected events, along with 5 additional events. The significance of $v_{e}$

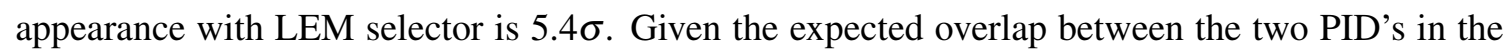
simulation, the trinomial probability of observing 11 events such that (LID and LEM) : (LEM, not LID) : (LID, not LEM) combination is 6:5:0, or less likely distributions, is $7.8 \%$. Figure 8 shows the LID and energy distribution of these 11 events. 

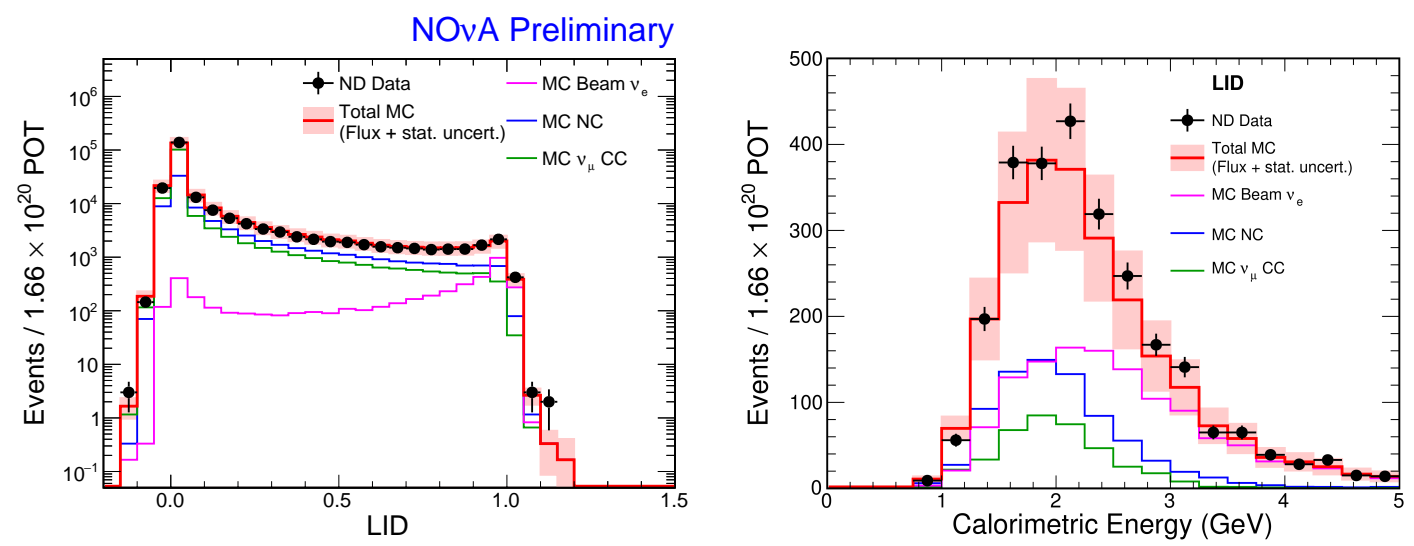

Figure 7: Left: Distribution of transverse momentum fraction for cosmic data and simulated $v_{e} \mathrm{CC}$ signal and beam backgrounds. Right: Rejection of cosmics at different cut levels in the FD, compared to the impact on the $v_{e}$ signal.
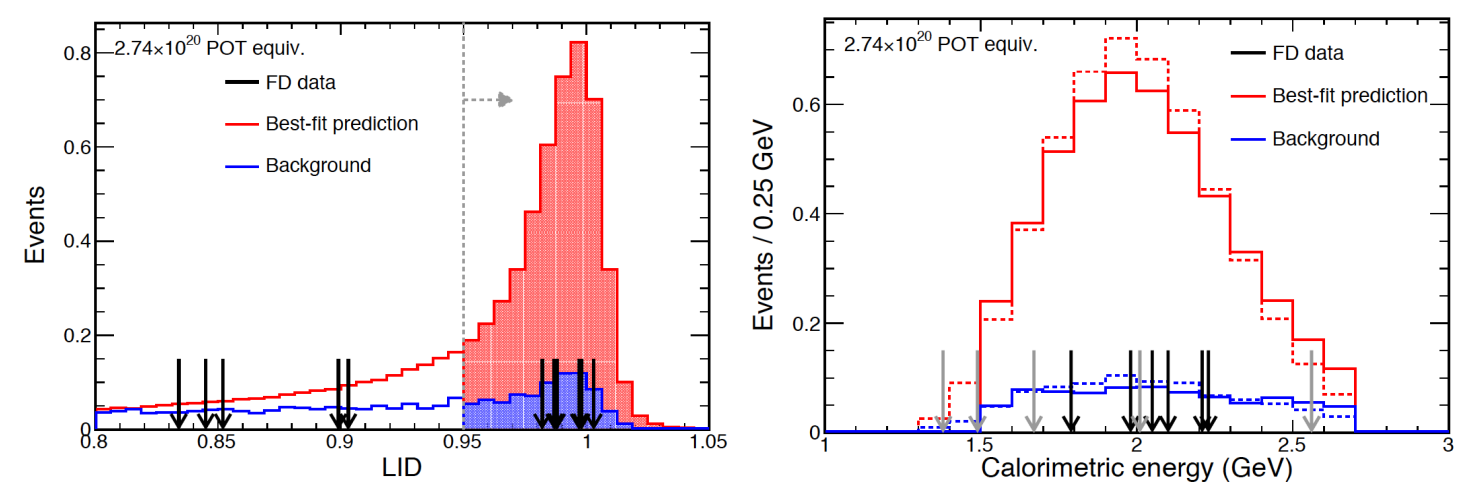

Figure 8: Left: LID distribution of 11 events selected by LID and LEM in the FD neutrino beam data. Right: The energy distribution of the 11 candidates.

These results are used to compute an allowed region in the $\sin ^{2} 2 \theta_{13}$ and $\delta_{C P}$ space, for normal and inverted hierarchy, as shown in Figure 10. The value of $\sin ^{2} \theta_{23}$ is assumed to be maximal, ie 0.5 and held fixed. To account for the effect of low statistics and physical boundaries, the Feldman and Cousins [14] statistical corrections are used. For the LID selector, the data are consistent with normal hierarchy within $1 \sigma$ for all values of $\delta_{C P}$. The larger event count observed by LEM argues for normal hierarchy, $\delta_{C P} \sim 3 \pi / 2$ with $\sin ^{2} \theta_{23}>0.5$.

Since the value of $\sin ^{2} \theta_{13}$ is well known from reactor neutrino oscillation experiments, this parameter is constrained at $0.086 \pm 0.005$ in the fit to test for the value of $\delta_{C P}$ most consistent with our observation for the two different hierarchies, while still holding $\sin ^{2} \theta_{23}$ fixed at maximal. These results are shown in Figure 9. The discontinuities are due to discrete set of possible event counts. The range of $0.1 \pi<\delta_{C P}<0.5 \pi$ for inverted hierarchy is disfavored at 90\% CL for LID. Since LEM selector selects more events, all values of $\delta_{C P}$ are disfavored at $90 \% \mathrm{CL}$ in inverted hierarchy, while in the normal hierarchy, $0.25 \pi<\delta_{C P}<0.95 \pi$ range is disfavored at $90 \%$ CL. These results have been reported in [15]. 


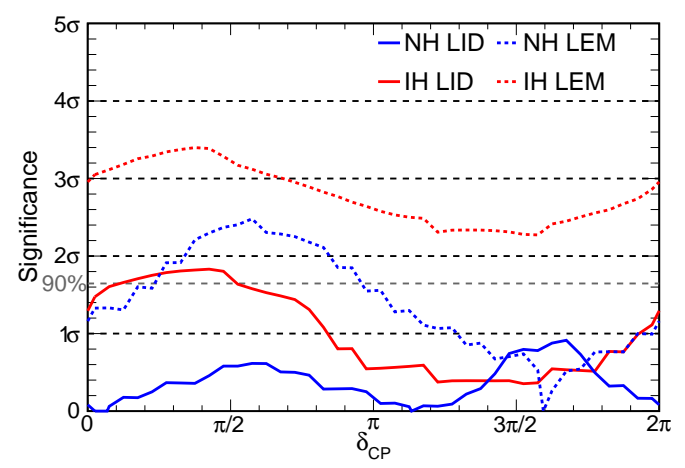

Figure 9: The significance at which values of CP violating phase $\delta$ are disfavored for the two hierarchies for LID and LEM. The value of $\sin ^{2} 2 \theta_{13}$ is constrained to the reactor values.
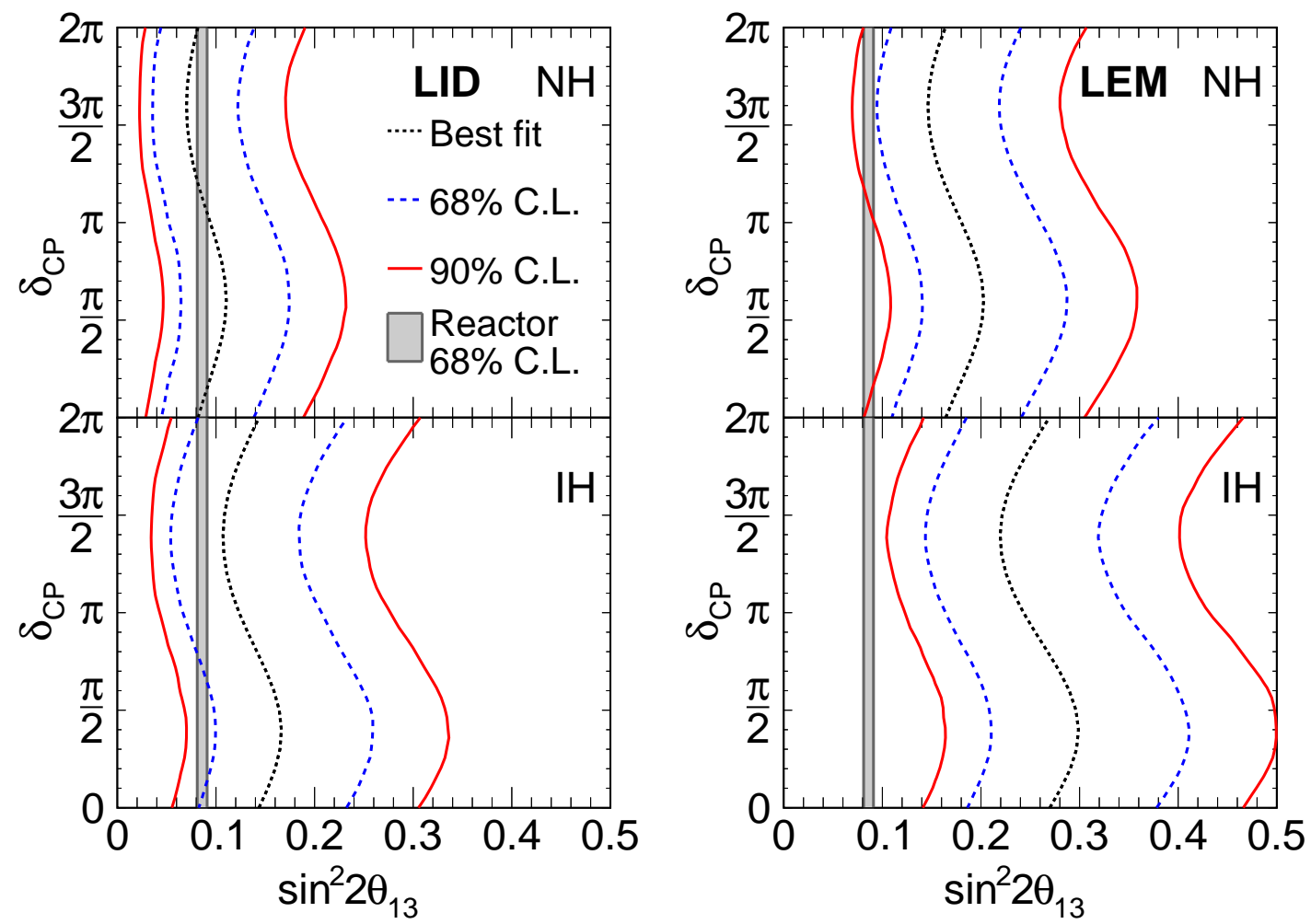

Figure 10: The $68 \%$ and $90 \% \mathrm{CL}$ allowed regions in the $\sin ^{2} 2 \theta_{13}$ and $\delta_{C P}$ space for LID and LEM. The upper panels are for normal hierarchy, while the lower panels are for inverted hierarchy.

\section{Conclusions}

With an exposure of $2.74 \times 10^{20}$ POT, the first oscillation results from the NOvA experiment have been reported. The measurement of the $v_{\mu}$ disappearance probability finds the best fit values that are consistent with maximal mixing, and have competitive sensitivity with other experiments. In the appearance channel, $6 v_{e}$ candidates on a background of $\sim 1$ event have been observed, a $3.3 \sigma$ excess. This observation disfavors the values of $\delta_{C P}$ around $\pi / 2$ for inverted hierarchy when 
reactor constraint is imposed on $\sin ^{2} 2 \theta_{13}$ and $\sin ^{2} \theta_{23}$ is held fixed at maximal.

NOvA is continuing to take data, and results with twice the exposure than reported here have been presented at recent conferences. In future, NOvA will measure the oscillation probabilities for anti-neutrinos, which will help disentangle some of the degeneracies in the oscillation parameters.

\section{References}

[1] B. Ananthanarayan and Ritesh K. Singh. Direct observation of neutrino oscillations at the sudbury neutrino observatory. Curr. Sci., 83:553, 2002, physics/0208096.

[2] Ashie et. al. Measurement of atmospheric neutrino oscillation parameters by super-kamiokande i. Phys. Rev. D, 71:112005, Jun 2005.

[3] P. Adamson et al. Combined analysis of $v_{\mu}$ disappearance and $v_{\mu} \rightarrow v_{e}$ appearance in minos using accelerator and atmospheric neutrinos. Phys.Rev.Lett., 112:191801, 2014, 1403.0867.

[4] F.P. An et al. Observation of electron-antineutrino disappearance at daya bay. Preprint, 2012.

[5] Kim Soo-Bong et al. Observation of reactor electron antineutrino disappearance in the reno experiment. 2012.

[6] Y. Abe et al. Reactor electron antineutrino disappearance in the double chooz experiment. Phys. Rev., D86:052008, 2012, 1207.6632.

[7] F. P. An et al. A new measurement of antineutrino oscillation with the full detector configuration at daya bay. Phys. Rev. Lett., 115:111802, 2015, 1505.03456.

[8] D.S. Ayres et al. The nova technical design report. Technical report, Fermilab, Batavia, Illinois, 2007.

[9] Alfredo Ferrari, Paola R. Sala, Alberto Fasso, and Johannes Ranft. FLUKA: A multi-particle transport code (Program version 2005). Technical report, 2005.

[10] S. Agostinelli et al. GEANT4: A Simulation toolkit. Nucl.Instrum.Meth., A506:250-303, 2003.

[11] C. Andreopoulos et al. The GENIE Neutrino Monte Carlo Generator. Nucl. Instrum. Meth., A614:87-104, 2010, 0905.2517.

[12] A Aurisano et al. The nova simulation chain. Submitted to Journal of Physics: Conference Series.

[13] P. Adamson et al. First measurement of muon-neutrino disappearance in nova. Phys. Rev. D, 93:051104, Mar 2016.

[14] Gary J. Feldman and Robert D. Cousins. Unified approach to the classical statistical analysis of small signals. Phys. Rev. D, 57:3873-3889, Apr 1998.

[15] P. Adamson et al. First measurement of electron neutrino appearance in nova. Phys. Rev. Lett., 116:151806, Apr 2016. 